\title{
Superior Mesenteric Artery Syndrome: A Rare Case Report
}

\author{
Dr. Md. Tabrez Aziz ${ }^{1}$, Dr. Md. Omar Tabrez ${ }^{2}$ \\ ${ }^{1,2}$ Department of Surgery, Rahmania Medical Centre, Motihari, Bihar, India
}

\begin{abstract}
Superior mesenteric artery (SMA) syndrome is an uncommon cause of proximal intestinal obstruction. Obstruction results from the vascular compression of the third portion of the duodenum between the aorta and the SMA. We present a case of SMA syndrome in a 20-year-old female presented with a 2 month history of nausea, vomiting, and abdominal pain. The diagnosis was established by Barium UGI series and CECT Abdomen. Conservative treatment didn't relieved so planned for Surgery. After surgery there was complete relief with no any complication.
\end{abstract}

Keywords: SMAS, SMA Syndrome, Cast syndrome, Willkie syndrome, Arteriomesenteric duodenal obstruction

\section{Introduction}

Superior mesenteric artery (SMA) syndrome is relatively rare aetiology of upper intestinal obstruction. It results from vascular compression of the third portion of the duodenum between the aorta and the SMA. The result is chronic, intermittent or acute obstruction of the duodenum, which may be either complete or partial. We present a case of SMA syndrome in a 20-year-old female who presented with a 2day history of nausea, postprandial vomiting, and abdominal pain.

\section{Case Report}

A 20 year young female admitted with the complaint of abdominal pain, epigastric fullness with vomiting and weight loss. Pain was colicky in nature since 2 months for which she was taking treatment from private practitioner. It was precipitating by eating food and relieved after bouts of vomiting (undigested food).

Her vitals were stable. Abdominal examination revealed epigastric fullness and hyper peristaltic bowel sounds. Routine blood and urine examination were normal. Monteux and erythrocyte sedimentation rate (ESR) test were negative. Ultrasonography (USG) of the abdomen was normal. Upper gastrointestinal barium study showed dilated stomach with dilated second part of the duodenum and cut off at the third part of duodenum. There was no relief of obstruction in the left lateral decubitus or prone position. Contrast enhanced computed tomography (CECT) showed distended stomach with dilatation of second part and constriction/extrinsic compression of the third part of duodenum between aorta and SMA (figure 1 and figure 2).

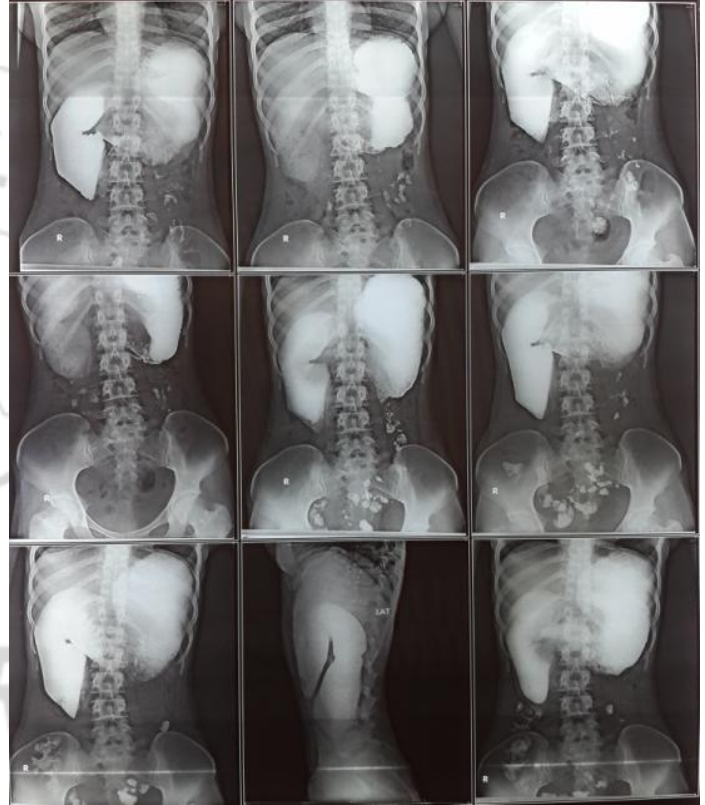

Figure 1: CECT abdomen showing dilated duodenum

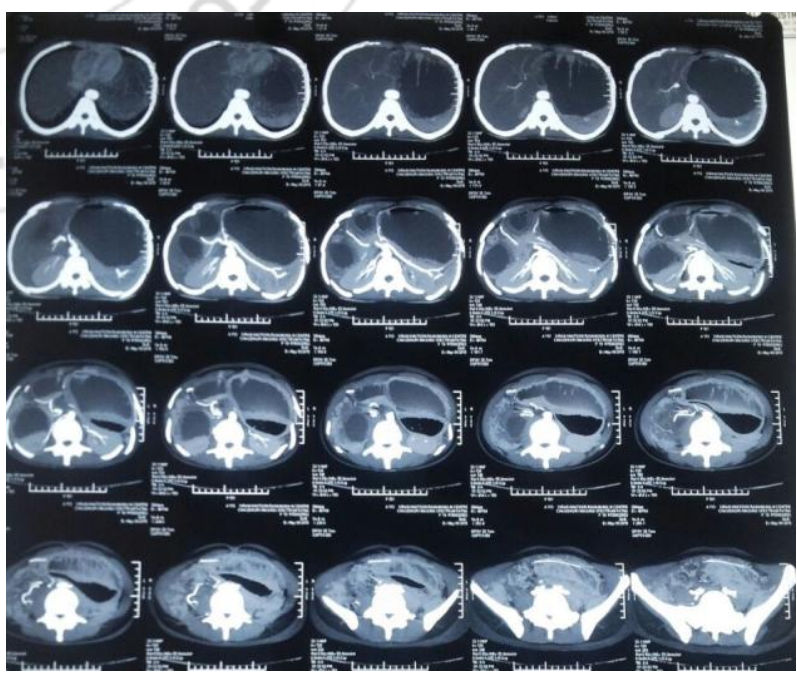

Figure 2: CECT abdomen showing dilated duodenum

The clinical symptoms and signs with investigative findings suggested the diagnosis of SMAS. Conservative treatment was tried for one month. The patient was told to take frequent small meals of nutritious liquid, advised to lie on left side/prone following meals. Metaclopramide was also 


\section{International Journal of Science and Research (IJSR) \\ ISSN (Online): 2319-7064 \\ Index Copernicus Value (2013): 6.14 | Impact Factor (2015): 6.391}

advised but no relief of symptoms found, so surgery was planned.

Exploratory laparotomy through a midline incision was done. Intra-operative findings confirmed the extrinsic obstruction of third part of duodenum with distension of $2^{\text {nd }}$ part. The site of obstruction was further confirmed by nasogastric airinsufflations. A retro colic duodeno-jejunostomy, side to side anastomosis done. In post-operative follow up, patient was symptom free and started taking normal diet and added some weight.

\section{Discussion}

Superior mesenteric artery (SMA) syndrome is an unusual form of intestinal obstruction caused by vascular compression of the third portion of the duodenum between the aorta and the SMA. This syndrome is often precipitated by conditions that narrow the aortomesentric angle, including possible immobilization, external compression due to body cast treatment of a spinal fracture, or rapid weight loss for any reason [1]. In previous reports, many other causes have also been suggested, such as a high insertion of the duodenum at the ligament of Treitz, a congenitally low origin of the SMA and compression of the duodenum caused by peritoneal adhesions after duodenal malrotation [4, 5]. The exact prevalence of the disorder in not well established; nonetheless, the estimated prevalence rates based on a gastrointestinal barium series range vary from $0.01 \%$ to 0.3 $\%$ [3]. SMA syndrome occurs in females more often than in males; some authors believe that SMA syndrome is not rare, but rather is a matter of degree with many people showing only mild compression not presenting with symptoms [6]. In the presence of vascular compression, a variety of gastrointestinal symptoms, including nausea, vomiting, postprandial abdominal pain and weight loss, may develop; these seem to occur when the aortomensnetric angle is less than $22^{\circ}$ and/or the distance is less than $8 \mathrm{~mm}$. Gastric and/or duodenal dilatation and a diminished SMA-aorta distance show a significant correlation with the clinical symptoms of SMA syndrome [7]. In our case, the aortomesentric angle was measured to be $18^{\circ}$ (normal: $25^{\circ}-60^{\circ}$ ) and the distance was measured as $4 \mathrm{~mm}$ (normal: $10-28 \mathrm{~mm}$ ). The patient presented with nausea, postprandial vomiting, and abdominal pain; these were improved when the patient underwent conservative treatment. Patients suffering from SMA syndrome usually find relief from the symptoms by assuming a left lateral decubitus, lying prone, or assuming a knees-tochest positioning, as was seen in the present case. The clinical diagnosis can be confirmed radiologically in 95\% of cases [8]. The following radiological criteria [8] have been established for the diagnosis of SMA: - dilatation of the first and second portions of the duodenum, with or without gastric dilatation; - abrupt vertical and oblique compression of the mucosal folds; - antiperistalsis flow of barium proximal to the obstruction, producing a to-and-fro movement; - delay of 4 to 6 hours in transit through the gastroduodenal region.

Ultrasonography has been shown to be able to measure the aortomensnetric distance accurately and may provide a useful alternative method of assessment in paediatric and obstetric populations [3, 9]. Conventional diagnostic evaluation begins with a Barium UGI series; multi-sliced CT scanning has been shown to be able to provide accurate radiographic findings and has the additional ability of be able to identify complications that may require immediate surgical intervention [10]. Once identified, usually cases respond well to conservative therapy, with surgical correction being reserved for chronic refractory cases[2,10], such as those with a long history of vomiting, those showing progressive weight loss, those with pronounced dilatation and those having stasis of the duodenum.

\section{Conclusion}

Literature review has suggested CECT abdomen is the definitive diagnosis. Conservative management can be tried though surgical management is the treatment of choice if no relief seen with the conservative management.

\section{References}

[1] Welsch T, Buchler MW, Kienle P. Recalling superior mesenteric artery syndrome. Dig Surg 2007;24:149-56.

[2] Raissi B, Taylor BM, Taves DH. Recurrent superior mesenteric artery (Wilkie's) syndrome: a case report. Can J Surg 1996;39:410-6.

[3] Ylinen P, Kinnunen J, Hockerstedt K. Superior mesenteric artery syndrome. A follow-up study of 16 operated patients. J Clin Gastroenterol 1989;11:386-91.

[4] Ortiz C, Cleveland RH, Blickman JG, et al. Familial superiormesenteric artery syndrome. Pediatr Radiol 1990;20:588-9.

[5] Wilkie DPD. Chronic duodenal ileus. Am J Med Sci 1927;173:643-9.

[6] Pleşa A, Constantinescu C, Crumpei F, Cotea E. Superior mesenteric artery syndrome: an unusual case of intestinal obstruction. J Gastrointestin Liver Dis 2006;15:69-72.

[7] Unal B, Aktas A, Kemal G, et al. Superior mesenteric artery syndrome: CT and ultrasonography findings. Diagn Interv Radiol 2005;11:90-5.

[8] Hearn JB. Duodenal ileus with special reference to superior mesenteric artery compression. Radiology 1966;86:305-10.

[9] Baltazar U, Dunn J, Floresguerra C, Schmidt L, Browder W. Superior mesenteric artery syndrome: an uncommon cause of intestinal obstruction. South Med J 2009;3:606-8.

[10]Lippl F, Hannig C, Weiss W, Allescher HD, Classen M, Kurjak M. Superior mesenteric artery syndrome: diagnosis and treatment from gastroenterologist's view. J Gastroenterol 2002;37:640-3. 